\title{
3D-Printed Prosthesis Replacement for Limb Salvage after Radical Resection of an Ameloblastoma in the Tibia with 1 Year of Follow Up: A Case Report
}

\author{
Dehong Feng, Junshan He, Chenyu Zhang, Ling Wang, Xiaofeng Gu, and Yu Guo \\ Department of Joint Surgery, The Affiliated Wuxi People's Hospital of Nanjing Medical University, Wuxi, Jiangsu, China.
}

\begin{abstract}
Ameloblastoma in the tibia is rare. Limb reconstruction after tumor resection is challenging in terms of selection of the operative method. Here, we report a case of radical resection of an ameloblastoma in the mid-distal tibia combined with limb salvage using a three-dimensional (3D)-printed prosthesis replacement, with 1-year follow-up results. After receiving local institutional review board approval, a titanium alloy prosthesis was designed using a computer and manufactured with 3D-printing technology. During the operation, the stem of the prosthesis was inserted closely into the proximal tibial medullary cavity. Then, the metal ankle mortise and the talus were compacted closely. Radiographic results at 1-year follow up showed that the prosthesis was well placed, and no loosening was observed. The Musculoskeletal Tumor Society (MSTS) 93 functional score was 26 points, and the functional recovery percentage was $86.7 \%$. Computer-assisted 3D-printing technology allowed for more volume and structural compatibility of the prosthesis, thereby ensuring a smooth operation and initial prosthetic stabilization. During the follow up, the presence of bone ingrowths on the porous surface of some segments of the prosthesis suggested good outcomes for long-term biological integration between the prosthesis and host bone.
\end{abstract}

Key Words: 3D-printing, tibia, ameloblastoma, prosthesis

\section{INTRODUCTION}

Ameloblastoma occurs rarely in long bones and is slightly more common in the tibia. ${ }^{1}$ Most reports comprise individual cases, among which the treatment methods have differed. ${ }^{2-8}$ Since 1993, many scholars have used customized tibia prostheses to reconstruct limbs after tumor resection. ${ }^{9-14}$ However, no cases of ameloblastoma were reported among these cases. Herein, we report a case of IB stage ameloblastoma in the middistal tibia and distal fibula of the right limb that was treated

\footnotetext{
Received: February 7, 2019 Revised: June 19, 2019

Accepted: July 9, 2019

Corresponding author: Dehong Feng, MD, Department of Joint Surgery, The Affiliated Wuxi People's Hospital of Nanjing Medical University, No.299 Qingyang Road, Nanchang District, Wuxi, Jiangsu 214023, China.

Tel: 86-0510-85350113, Fax: 86-0510-82702233, E-mail: fengdh_wuxiph@sina.cn

-The authors have no potential conflicts of interest to disclose.

(c) Copyright: Yonsei University College of Medicine 2019

This is an Open Access article distributed under the terms of the Creative Commons Attribution Non-Commercial License (https://creativecommons.org/licenses/ by-nc/4.0) which permits unrestricted non-commercial use, distribution, and reproduction in any medium, provided the original work is properly cited.
}

with tumor radical resection combined with implanted composite of mid-distal tibial three-dimensional (3D)-printing prosthesis and allogenic tibial segment implantation. The study was approved by the institutional review board of the Affiliated Wuxi People's Hospital of Nanjing Medical University [IRB No: 2017-(01)]. Informed consent was obtained from the patient for the operation.

\section{CASE REPORT}

A 40-year-old man presented with right crus pain for a month. Magnetic resonance imaging showed mixed signals in the mid-distal segment of the right tibia and the distal part of the fibula, with low and high signals on T1- and T2-weighted images, respectively (Fig. 1). Tibial lesions penetrated the cortex, and puncture biopsy revealed ameloblastoma. Enneking stage was IB (G1, T2, and M0).

\section{Implant customization}

In a computer, 3D images of the tibia were reconstructed with 

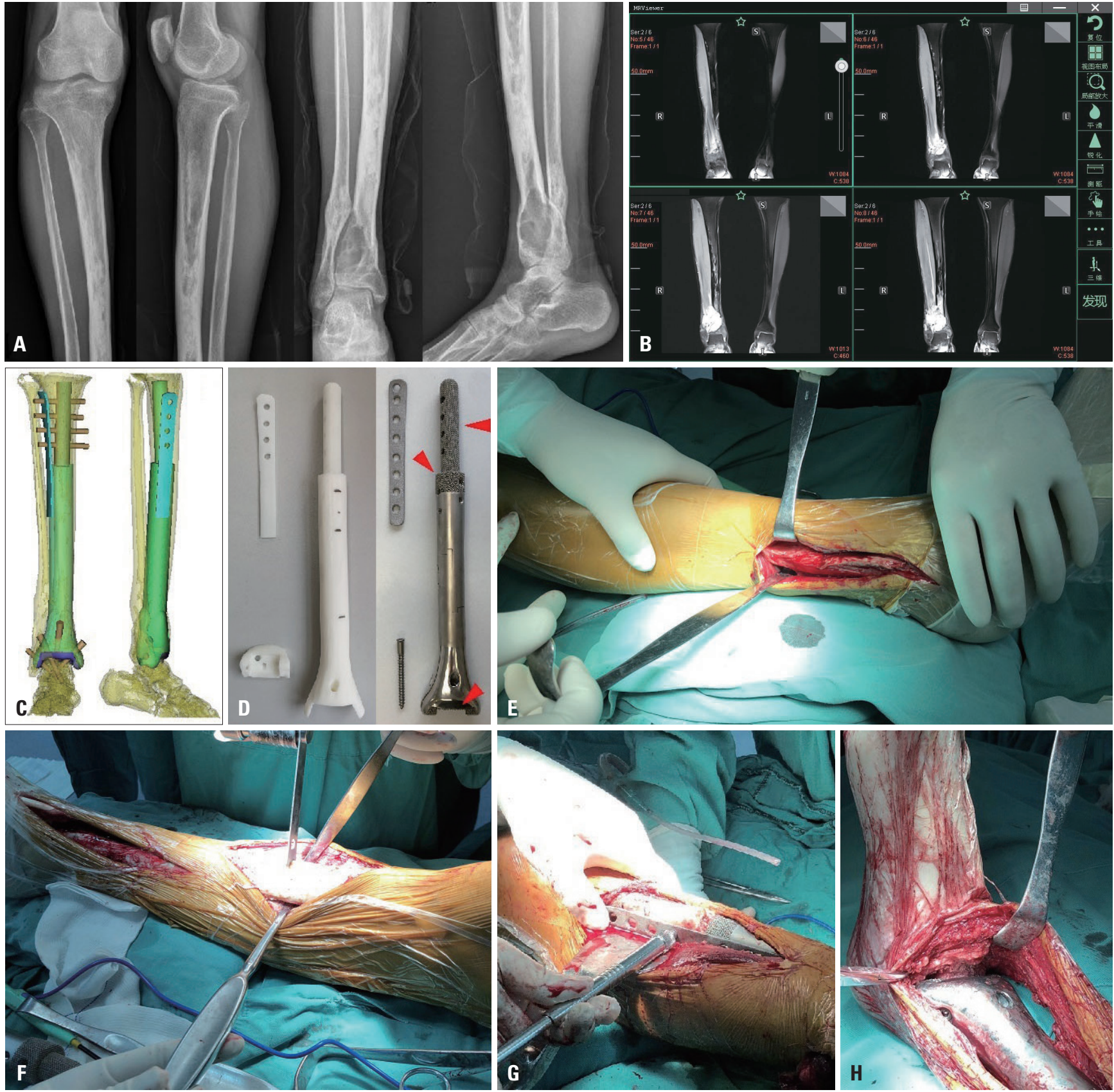

Fig. 1. Preoperative examination, design, and construction of the prosthesis using three-dimensional (3D)-printing technology. (A) Radiography of diseased tibia. (B) Magnetic resonance imaging of the diseased tibia and fibula. (C) 3D prosthesis design in a computer. (D) 3D-printing resinous model and titanium alloy prosthesis; the latter has a porous printed structure $(0.25-0.40 \mathrm{~cm}$ thickness) of the bone trabecula on the peripheral surface of the stem, collar, and ankle mortise (red arrowheads). The prosthesis was made with 3D printing in one step with its porous coating. (E) Incision I. (F) Incision II and incision III. (G) Installation of the proximal end of the prosthesis. (H) Installation of the distal end of the prosthesis.

reverse data of computed tomography (CT) of 0.625-mm slice thickness. The digital image of the prosthesis was designed according to the predicted range of radical resection. Then, the resinous tibia and prosthesis were printed with Lite 450 HD (Shanghai Union 3D Technology Co., Ltd., Shanghai, China) at a 1:1 size for the installation test. After debugging, a titanium alloy (Ti-6Al-4V; AP\&C Advanced Powder and Coatings Inc., Boisbriand, Quebec, Canada) prosthesis was printed in one step with its porous coating with Arcam Q10 (Arcam AB, Mölndal, Sweden) by Beijing Chunlizhengda Medical Instruments Company. The aperture, wire diameter, and porosity of the porous coating were $0.40-0.50,0.40-0.45$, and $68-78 \%$, respectively. The prosthetic cylindrical body was $2.6 \mathrm{~cm}$ in diameter and $23.5 \mathrm{~cm}$ in length, and could be split in the middle. The stem was $1.8 \mathrm{~cm}$ in diameter and $9.5 \mathrm{~cm}$ in length. The keyhole was equipped with an interlocking screw with a steel plate. 


\section{Surgical procedures}

After general endotracheal anesthesia, a $12.0-\mathrm{cm}$ long incision (I) was performed on the surface of the distal fibula. The fibula was amputated at $3.0 \mathrm{~cm}$ above the lesion. Then, a 18.0$\mathrm{cm}$ long incision (II) was made on the surface of the mid-distal tibia, which included a needle path for biopsy. At last, a 15.0$\mathrm{cm}$ long incision (III) on the anterior surface of the middle tibia was performed, and the tibia was amputated with stepsection at $3.0 \mathrm{~cm}$ above the lesion. The diseased bone and surrounding soft tissues were removed completely at its margin, and no residual tumor tissue was found in the frozen-section examination during the operation. The tibial stump was repaired with allogenic tibia to restore its intact structure. After directional reaming with a $1.6-\mathrm{cm}$ diameter reamer, the prosthetic stem with a porous surface was inserted into the medullary cavity, and then fixed with a plate and interlocking screw. Next, the metal ankle mortise and the osteotomic cancellous bone of the talus were also compacted closely and fixed with a number of screws. Finally, two parts of the prosthesis were connected with screws.

\section{Surgical outcome}

There were no injuries to vessels and nerves. The patient was able to walk with a single crutch after 2 weeks, and he was told to wear a brace when walking after 8 weeks. At 12 weeks, the brace was removed. The limb function was excellent at 1 year after surgery, and the MSTS 93 score was $86.7 \%(26 / 30) .{ }^{15}$ The ranges of movement were $0^{\circ}$ (extension) to $130^{\circ}$ (flexion) in the knee joint, and $15^{\circ}$ (dorsal extension) to $10^{\circ}$ (plantar flexion) in the mid-tarsal joint (Table 1). CT and radiography showed no signs of prosthesis loosening (partial regenerated bone ingrowth around the prosthesis and no progressive radiolucent zone) (Fig. 2). No tumor recurrence and metastasis were found.

\section{DISCUSSION}

The first type of tibial prostheses with an ankle joint was hingerestricted or semi-constrained..$^{9-12,14}$ The main complications were talus collapse, prosthesis loosening, fibula impact, incision necrosis, deep infection, and tumor recurrence. Although these scholars all believed that a tibial prosthesis with an ankle joint can achieve better short-term function with limb reconstruction than with other methods, such as bone transplantation and tumor bone replantation after inactivation, they also emphasized the need for strict selection of cases before opera- tion in view of more complications and poor late outcomes in some cases. ${ }^{9-12,14}$

Another type of prosthesis has no joints. Economopoulos, et al. ${ }^{13}$ reported a case of giant cell tumor of the distal tibia that was repaired with porous tantalum metal prosthesis without a joint. At 5 years after the operation, the patient had no pain when walking, and the mid-tarsal joint had $5^{\circ}$ of dorsal extension and $10^{\circ}$ of plantar flexion. They believed that the life expectancy of jointless prosthesis is better, although the tibiotalar joint will have lost its mobility. Also, the simpler surgical process in the operation is another advantage of jointless prosthesis.

In this case, ameloblastoma has a wide range of lesions, accounting for more than $75 \%$ of the total length of the tibia. If the bone of the diseased limb is reconstructed with a large segment of allograft, the bone regeneration will be slow, and the possibility of complications, such as allograft rejection, fracture, and nonunion, will increase. As mentioned above, local stress to a hinged prosthesis will be more intense and complications, such as loosening and talus collapse, will occur more readily. ${ }^{11,16}$ There are still complications, such as talus prosthesis loosening, after the use of a semi-constrained artificial ankle. ${ }^{10-12} \mathrm{As}$ in the present case, the distal fibular tumor segment often cannot be retained, and it can be very difficult to reconstruct the peri-ankle ligaments between the metal prosthesis and the host skeleton. Therefore, a mid-distal tibial prosthesis without an ankle joint should be used for limb reconstruction. Also, the initial stability of the prosthesis was beneficial for early initiation of rehabilitation, which is an advantage of this type of limb salvage, compared with allograft bone transplantation.

It has been reported that postoperative incision necrosis and infection are not uncommon during the operation of tibial tumors. ${ }^{9-14}$ In the present case, the lesion was extensive. To prevent skin necrosis after the operation, three incisions were designed. All were within $20 \mathrm{~cm}$ in length and staggered with each other. Incision I was $7.0 \mathrm{~cm}$ away from incision II. Likewise, the distal end of incision III and the proximal end of incision II were also $4.5 \mathrm{~cm}$ apart.

In the present case, a more intuitive understanding and accurate data of the diseased tibia could be obtained using a computer and resin model before the operation. Thereby, the most feasible schemes were devised during the manufacturing and implantation of the personalized prosthesis. When using the computer to simulate the surgery, we discovered that the remaining tibia may not be able to grasp the prosthetic stem according to the criteria of osteotomy. Thus, an allogenic tibia was prefabricated for bone grafting.

Table 1. MSTS 93 Scores of Surgical Limbs at 6 and 12 Months after Operation

\begin{tabular}{lcccccc}
\hline \multicolumn{1}{c}{ Time } & Pain & Function & Emotional acceptance & Support & Walking & Gait \\
\hline 6 months after operation & 5 & 3 & 4 & $3^{*}$ & 3 & $1^{\dagger}$ \\
12 months after operation & 5 & 3 & 5 & 5 & 5 & 3 \\
\hline
\end{tabular}

The MSTS 1993 score includes pain, function, emotional acceptance, supports, walking ability, and gait, and ranges from 0 to 30 . Excellent: 24-30 points, good: 18-23 points, fair:12-17 points, poor: 12 points or less. The final MSTS 1993 score is presented as a percentage.

${ }^{*}$ Brace using; ${ }^{\dagger}$ Major cosmetic. 

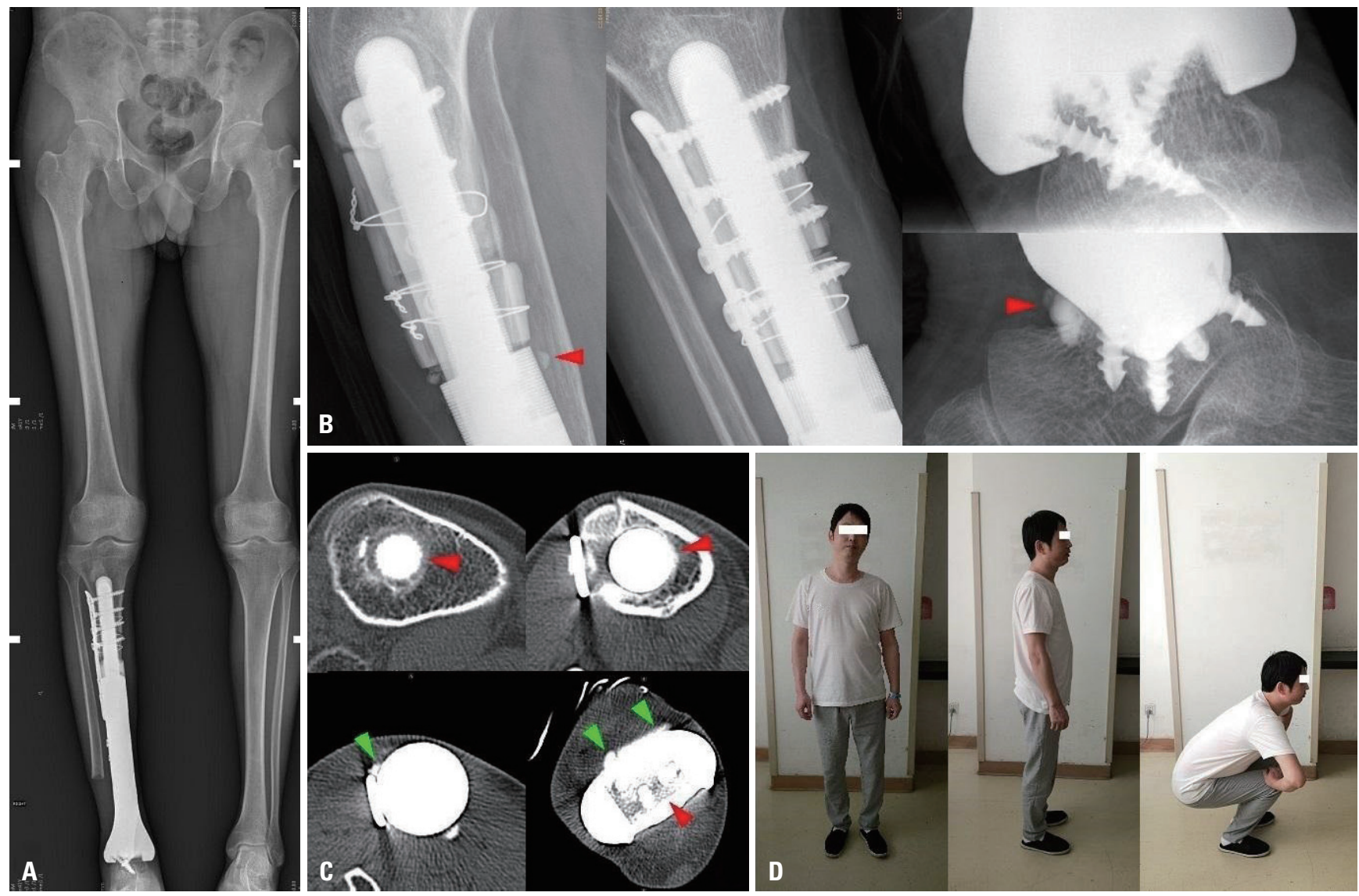

Fig. 2. Postoperative review. (A) Good prosthesis position and lower limb force line in full-length radiography images at 3 months after the operation. (B) At 12 months after the operation, radiography images show no obvious progressive radiolucent zones around the prosthesis stem and metal ankle mortise, as well as several new bone bridge spanning the bone-prosthesis junction outside the cortex and prosthesis (red arrowheads). (C) Computed tomography scan shows bone growth into the porous surface of the prosthetic stem and ankle mortise (red arrowheads) and into the porous surface of the prosthetic collar and the front of metal ankle mortise (from the extracortical bone bridge, green arrowheads) at 9 months after the operation. (D) Lower extremity movement of the patient at 9 months after the operation.

Hollander, et al. ${ }^{17}$ reported that after co-culture of porous titanium alloy scaffolds with human osteoblasts in vitro for 2 weeks, a large number of osteoblasts adhered to and proliferated in the surface and pores of the scaffolds. In this case, CT scan showed that the regenerated bone of the host had grown into the porous surface of the prosthetic stem and ankle mortise at 9 months after operation. The new extra-cortical bone bridges connected the tibial stump to the bone-prosthesis junction. Meanwhile, the new bone bridge spanned the junction of the talus and prosthesis in the anterior side of the talus. For 12 consecutive months after operation, radiography showed no progressive radiolucent zones around the prosthetic stem and metal ankle mortise, suggesting no obvious loosening of the prosthesis.

In this case, the application of 3D-printing technology to construct a prosthesis replacement achieved good short-term efficacy. We believe that the trabecular porous structure design will result in a good outcome for the long-term biological fixation of the prosthesis.

\section{AUTHOR CONTRIBUTIONS}

Conceptualization: Dehong Feng. Data curation: Yu Guo and Ling Wang. Formal analysis: Ling Wang and Yu Guo. Funding acquisition: Dehong Feng. Investigation: Junshan He and Chenyu Zhang. Methodology: Ling Wang and Xiaofeng Gu. Project administration: Dehong Feng and Xiaofeng Gu. Resources: Dehong Feng, Junshan He, and Xiaofeng Gu. Software: Chenyu Zhang and Yu Guo. Supervision: Ling Wang and Xiaofeng Gu. Validation: Chenyu Zhang and Ling Wang. Visualization: Junshan He. Writing_original draft: Dehong Feng and Junshan He. Writing_review \& editing: Dehong Feng.

\section{ORCID iDs}

Dehong Feng

Junshan He Chenyu Zhang

Ling Wang

Xiaofeng $\mathrm{Gu}$

Yu Guo https://orcid.org/0000-0003-3979-3442 https://orcid.org/0000-0003-0731-6238 https://orcid.org/0000-0003-0049-4234 https://orcid.org/0000-0002-2680-3624 https://orcid.org/0000-0003-1286-3105 https://orcid.org/0000-0002-7461-2822 


\section{REFERENCES}

1. Huvos AG, Marcove RC. Adamantinoma of long bones. A clinicopathological study of fourteen cases with vascular origin suggested. J Bone Joint Surg Am 1975;57:148-54.

2. Perez-Atayde AR, Kozakewich HP, Vawter GF. Adamantinoma of the tibia. An ultrastructural and immunohistochemical study. Cancer 1985;55:1015-23.

3. Kim JY, Kang GH, Chi JG. Adamantinoma of tibia with predominant features of fibrous dysplasia-a case report. J Korean Med Sci 1996;11:444-8.

4. Satyanarayana S, Jawed KZ, Sirohi D, Sikdar J. Adamantinoma of tibia. Med J Armed Forces India 2002;58:348-9.

5. Ramesh R, Burrah R, Thambuchetty N, Shivakumar K, Ananthamurthy A, Manjunath S. Adamantinoma of the tibia: a case report. Indian J Surg Oncol 2012;3:239-41.

6. Anoumou MN, Kouameu M, Koffi EK, Varango G. Adamantinoma of the tibia: a case report. J Orthop Case Rep 2014;4:15-7.

7. Giannoulis DK, Gantsos A, Giotis D, Paschos NK, Vagionas A, Arnaoutoglou CM, et al. Multiple recurrences and late metastasis of adamantinoma in the tibia: a case report. J Orthop Surg (Hong Kong) 2014;22:420-2.

8. Zhao Z, Zhang X, Yu G. A case of tibial ameloblastoma. Chinese Journal of Repair and Reconstruction Surgery 2016;30:129.

9. Lee SH, Kim HS, Park YB, Rhie TY, Lee HK. Prosthetic reconstruction for tumours of the distal tibia and fibula. J Bone Joint Surg Br 1999;81:803-7.

10. Abudu A, Grimer RJ, Tillman RM, Carter SR. Endoprosthetic re- placement of the distal tibia and ankle joint for aggressive bone tumours. Int Orthop 1999;23:291-4.

11. Natarajan MV, Annamalai K, Williams S, Selvaraj R, Rajagopal TS. Limb salvage in distal tibial osteosarcoma using a custom mega prosthesis. Int Orthop 2000;24:282-4.

12. Shekkeris AS, Hanna SA, Sewell MD, Spiegelberg BG, Aston WJ, Blunn GW, et al. Endoprosthetic reconstruction of the distal tibia and ankle joint after resection of primary bone tumours. J Bone Joint Surg Br 2009;91:1378-82.

13. Economopoulos K, Barker L, Beauchamp C, Claridge R. Case report: reconstruction of the distal tibia with porous tantalum spacer after resection for giant cell tumor. Clin Orthop Relat Res 2010; 468:1697-701.

14. Ajit Singh V, Nasirudin N, Bernatt M. Endoprosthetic reconstruction for giant cell tumors of the distal tibia: a short term review. Asia Pac J Clin Oncol 2013;9:182-9.

15. Enneking WF, Dunham W, Gebhardt MC, Malawar M, Pritchard DJ. A system for the functional evaluation of reconstructive procedures after surgical treatment of tumors of the musculoskeletal system. Clin Orthop Relat Res 1993;(286):241-6.

16. Xiao $\mathrm{H}, \mathrm{Niu} \mathrm{X}$. Aseptic loosening rate and related factors of tumorous artificial joint prosthesis. Chinese Journal of Bone and Joint 2014;3:365-9.

17. Hollander DA, von Walter M, Wirtz T, Sellei R, Schmidt-Rohlfing B, Paar O, et al. Structural, mechanical and in vitro characterization of individually structured Ti-6Al-4V produced by direct laser forming. Biomaterials 2006;27:955-63. 